\title{
Time-varying serum gradient of hepatitis B surface antigen predicts risk of relapses after off-NA therapy
}

\author{
Nai-Hsuan Chien ${ }^{1,2,12}$, Yen-Tsung Huang ${ }^{3}$, Chun-Ying Wu ${ }^{4,5}$, Chi-Yang Chang ${ }^{2,6,10}$, Ming-Shiang Wu', \\ Jia-Horng Kao ${ }^{7,8}$, Lein-Ray Mo ${ }^{9}$, Chi-Ming Tai ${ }^{10}$, Chih-Wen Lin ${ }^{10}$, Tzeng-Huey Yang ${ }^{11}$, Jaw-Town Lin ${ }^{2,6,10}$ \\ and Yao-Chun $\mathrm{Hsu}^{2,6,10,13^{*}}$
}

\begin{abstract}
Background: The serum gradient of hepatitis B surface antigen (HBsAg) varies over time after cessation of nucleos(t) ide analog (NA) treatment in patients with chronic hepatitis B (CHB). The association between the time-varying HBsAg serum gradient and risk of relapse has not been elucidated.

Methods: This multicenter cohort study prospectively enrolled CHB patients who discontinued 3 year-NA treatment. Eligible patients were serologically negative for HBeAg and viral DNA at NA cessation. The participants $(n=140)$ were followed every 3 months through HBsAg quantification. Virological and clinical relapses were defined as viral DNA levels $>2000 \mathrm{IU} / \mathrm{mL}$ and alanine aminotransferase (ALT) levels $>80 \mathrm{U} / \mathrm{mL}$, respectively. The association of time-varying HBsAg levels with relapses was assessed through a time-dependent Cox analysis.

Results: During a median follow-up of 19.9 (interquartile range [IQR], 10.6-25.3) months, virological and clinical relapses occurred in 94 and 49 patients, with a 2-year cumulative incidence of 79.2\% (95\% confidence interval [CI], 70.9\%-86.4\%) and $42.9 \%(95 \% \mathrm{Cl}, 34.1 \%-52.8 \%)$, respectively. The serum level of HBsAg was associated with virological $(P<0.001)$ and clinical $(P=0.01)$ relapses in a dose-response manner, with adjusted hazard ratios of $2.10(95 \% \mathrm{Cl}, 1.45-3.04)$ and 2.32 ( $95 \% \mathrm{Cl}, 1.28-4.21)$. Among the patients $(n=19)$ whose HBsAg levels ever dropped below $10 \mathrm{lU} / \mathrm{mL}$, only one and three patients subsequently developed clinical and virological relapses.
\end{abstract}

Conclusion: The serum gradient of HBsAg measured throughout the off-therapy observation is associated with the subsequent occurrence of virological and clinical relapses in CHB patients who discontinue NA treatment.

Keywords: Chronic hepatitis B, Nucleos(t)ide analogs, Hepatitis B surface antigen quantification, Timedependent Cox proportion hazards model

\section{Background}

Chronic infection with hepatitis B virus (HBV) is the leading cause of liver-related morbidity and mortality worldwide, particularly in Asian countries [1]. Management of patients with chronic hepatitis B (CHB) has reached an era of antiviral therapy, with approved regimens consisting of interferon alpha and nucleos(t)ide analogs (NAs) [2-5]. Through the effective inhibition of viral replication, NAs not only

\footnotetext{
* Correspondence: gatsbyhsu@yahoo.com.tw; holdenhsu@gmail.com ${ }^{2}$ School of Medicine, Fu Jen Catholic University, New Taipei, Taiwan ${ }^{6}$ Division of Gastroenterology, Fu-Jen Catholic University Hospital, New Taipei, Taiwan

Full list of author information is available at the end of the article
}

ameliorate viremia and reduce hepatic inflammation, but also may prevent and even reverse liver fibrosis [6-8]. A large body of evidence corroborates the effectiveness of NAs in improving clinical outcomes $[9,10]$. However, off-therapy durability after discontinuation of NA treatment is typically unsustainable [11-19].

Because of high off-therapy relapse rates, major international guidelines currently recommend an indefinite prolongation of NA therapy, possibly until loss of hepatitis B surface antigen (HBsAg) with or without appearance of accompanying antibodies [12]. However, this strategy entails life-long treatment for most treated patients [13] and is not affordable in regions with 
resource-constrained health care systems, where, ironically, CHB is most prevalent [14]. Therefore, it is important to find out some factors to predict the risk of off-therapy relapse. Recently, intense research has been carried out to clarify predictors of off-therapy relapse and identify patients who maintain remission without resuming medication [17-22].

In response to the challenges associated with the safe discontinuation of NAs, we prospectively followed a multicenter cohort who discontinued NAs after a minimum of 3 years on therapy. We observed that the serum gradient of HBsAg observed at the end of treatment (EOT), in addition to serum alanine aminotransferase (ALT) levels and age, stratified the risks of both virological and clinical relapses [20]. After the cessation of NA therapy, the level of HBsAg may change over time, but its clinical implication has not been elucidated. Therefore, we conducted this study to explore the association between relapse risks and time-varying serum gradients of $\mathrm{HBsAg}$ measured during an off-therapy follow-up.

\section{Methods}

\section{Design and setting}

This prospective cohort study was conducted in three different regional teaching hospitals (E-Da Hospital, Kaohsiung, Lotung Poh-Ai Hospital, Yilan, and National Taiwan University Hospital Yun-Lin Branch, Yunlin) in Taiwan. Institutional review boards approved the study protocol (EMRP100-049) in all hospitals for patient recruitment and database establishment. Data analysis specifically for this study was also approved (EMRP-104082). Written informed consent was obtained from all participants prior to their enrollment.

\section{Study participants}

We consecutively screened adult patients with $\mathrm{CHB}$ who were going to discontinue NA therapy between July 1, 2011 and April 1, 2015, and we evaluated their eligibility. Patients were included if they had been diagnosed with $\mathrm{CHB}$ for at least 6 months prior to NA treatment, continuously received any NA (lamivudine, adefovir, telbivudine, entecavir, or tenofovir) for at least 3 years, were serologically negative for $\mathrm{HBeAg}$, and showed undetectable levels of HBV DNA at the end of NA therapy. Patients were excluded in the presence of coinfection with human immunodeficiency virus or hepatitis $\mathrm{C}$ virus, any malignancy, liver cirrhosis, hepatic encephalopathy, variceal hemorrhage, organ transplantation, previous use of interferon alpha for 1 month or longer, and concurrent use of cytotoxic or immunosuppressive medication. The diagnosis of liver cirrhosis was based on pathological proof or clinical criteria that included splenomegaly or esophagogastric varices in addition to typical sonographic features.
Patients had to discontinue NAs because of the national health insurance policy. Details of the reimbursement regulations have been previously reported. ${ }^{10}$ In brief, the therapeutic duration was principally restricted to 3 years among general patients without cirrhosis. Those who experienced $\mathrm{HBeAg}$ seroconversion on therapy were entitled to treatment consolidation for an additional 1 year.

\section{Follow-up after cessation of NAs}

Pertinent demographic, biochemical, serological, and virological data were collected at enrollment. After discontinuation of NAs, patients were monitored at a close interval of 3 months. The patients underwent physical checkup and laboratory measurement at each follow-up visit. They also underwent abdominal sonography along with serum alpha-fetoprotein estimation tests every 6 months for the surveillance of liver cancer.

Standardized quantification of serum HBsAg and viral DNA was carried out in the Taipei Pathology Institutes (Taipei, Taiwan). Serum HBsAg levels were measured through an automated immunoassay (Abbott Architect i2000, Abbott Park, IL, USA). Samples with HBsAg levels exceeding the upper limit of automatic detection $(250 \mathrm{IU} / \mathrm{mL})$ were manually diluted before quantification. Serum HBV DNA was quantified through a commercialized polymerase chain reaction method (COBAS TaqMan HBV Test, version 2.0, Roche Molecular Systems, Inc., Branchburg, NJ, USA) with a detection range of $20-1.7 \times 10^{8} \mathrm{IU} / \mathrm{mL}$.

\section{Definitions of virological and clinical relapses}

Virological relapse was defined as the reappearance of $>2000 \mathrm{IU} / \mathrm{mL}$ HBV DNA in serum. Clinical relapse was defined as an episode of elevated ALT $(>80 \mathrm{IU} / \mathrm{mL},>2$ times the normal conventional upper limit) and $>2000 \mathrm{IU} / \mathrm{mL}$ HBV DNA. Patients did not resume antiviral therapy until clinical hepatitis persisted for 3 months or longer, unless a risk of hepatic decompensation (serum bilirubin $>2 \mathrm{mg} / \mathrm{dL}$ or prothrombin time prolonged $>3 \mathrm{~s}$ ) was observed.

\section{Data analyses and statistical methods}

Continuous and categorical variables were summarized using the median and interquartile range (IQR) and proportion with exact numbers, respectively. The incidence rates of virological and clinical relapses were estimated using the Kaplan-Meier method. In a multivariate-adjusted Cox proportional hazards model for off-therapy relapses, the serum level of $\mathrm{HBsAg}$ was a time-varying variable that denoted each measurement after NA cessation. The dose-response relationship for the association between $\mathrm{HBsAg}$ levels and off-therapy relapses was illustrated by penalized splines in the Cox model. The results were reported as hazard ratios along with $95 \%$ confidence intervals 
(CIs). Data were analyzed using commercial software (Stata, version 13.0; Stata Corp, College Station, TX, USA). All statistical analyses were two-sided with significance set at $P<0.05$.

\section{Results}

Baseline characteristics of the participants

We monitored a total of 140 patients who discontinued NA therapy after a minimum of 3 years (median, 36.6; IQR, 36.4-37.0 months) between July 1, 2011 and April 1, 2015 (Fig. 1). They were followed for a median of 19.9 (IQR, 10.6-25.3) months following the cessation of NA therapy. The mean time interval between initial HBsAg level and last HBsAg level is $19.15 \pm 11$ months. Table 1 presents a summary of the characteristics of this study population, which was predominantly male (77.9\%, $n=109$ ) with a median age of 49.1 (IQR, 39.2-57.5) years. Prior to antiviral treatment, 39 patients were initially HBeAg-positive. They were all serologically negative for $\mathrm{HBeAg}$ after treatment and had been consolidated for at least 1 year (median, 18.2; IQR, 12.2-25.4 months) following $\mathrm{HBeAg}$ loss. The median levels of HBsAg, ALT, and alpha-fetoprotein

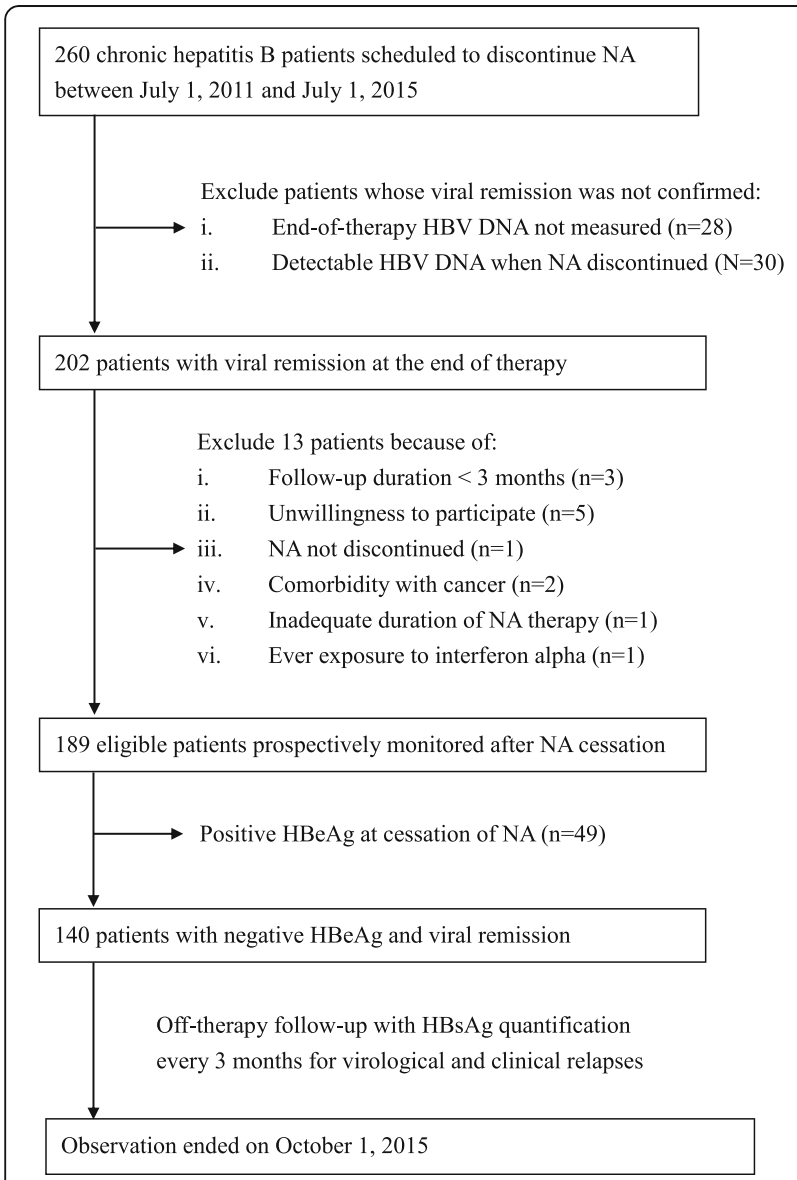

Fig. 1 The flowchart for the identification and enrollment of participants
Table 1 Characteristics of the study patients

\begin{tabular}{ll}
\hline Characteristics & All patients $(\mathrm{N}=140)$ \\
\hline Male gender, $n(\%)$ & $109(77.9 \%)$ \\
EOT Age, years & $49.1(39.2,57.5)$ \\
EOT anti-HBe-positive, $n(\%)$ & $131(93.6 \%)$ \\
EOT HBsAg, log IU/mL & $2.79(2.13,3.12)$ \\
EOT ALT, U/L & $22(16.5,34)$ \\
EOT AFP, ng/ml & $2.7(1.97,3.41)$ \\
Pretreatment HBeAg-positive, $n(\%)$ & $39(27.9 \%)$ \\
Pretreatment anti-HBe-positive, $n(\%)$ & $100(71.4 \%)$ \\
Pretreatment viral DNA, log IU/ml & $6.21(4.53,7.67)$ \\
Pretreatment ALT, U/L & $154(95,451)$ \\
On-therapy duration, month & $36.6(36.4,37.0)$ \\
Off-therapy follow-up ${ }^{a}$, month & $19.9(10.6,25.3)$ \\
Patients received Entecavir & $125(89.3 \%)$ \\
Patients received Tenofovir, & $9(6.4 \%)$ \\
Patients received Lamivudine or Telbivudine & $6(4.2 \%)$
\end{tabular}

Notes. ${ }^{\text {a }}$ patients were followed up until reuse of antiviral therapy; AFP alphafetoprotein, ALT Alanine transaminase, EOT end-of-therapy, HBeAg hepatitis B e antigen, $H B s A g$ hepatitis B surface antigen

were 2.79 (IQR, 2.13-3.12) $\log \mathrm{IU} / \mathrm{mL}, 22$ (IQR, 16.5-34) $\mathrm{U} / \mathrm{L}$, and 2.7 (IQR, 1.97-3.41) ng/mL at the end of treatment, respectively.

Clinical and virological relapses after NA discontinuation During the off-therapy follow-up, 49 and 94 participants developed clinical and virological relapses, respectively. The cumulative incidence rates of clinical relapses were $28.4 \%(95 \%$ CI, 21.2\%-37.5\%) and $42.9 \%$ (95\% CI, $34.1 \%-52.8 \%)$ at 1 and 2 years, respectively, and those of virological relapses were $62.0 \%$ (95\% CI, 53.5\%-70.6\%) and $79.2 \%(95 \% \mathrm{CI}, 70.9 \%-86.4 \%)$ at 1 and 2 years, respectively (Table 2). Seven patients with previous $\mathrm{HBeAg}$ positive experienced reoccurrence of $\mathrm{HBeAg}$. Twenty one patients had been treated with NA after relapse. No patient is complicated with hepatic decompensation after secession of NAs therapy in this study.

\section{Dose-response relationship of the time-varying $\mathrm{HBsAg}$ level and off-therapy relapses}

The penalized splines in the univariate Cox model characterized the dose-response curves between increments

Table 2 Clinical and virological relapses after cessation of nucleos(t)ide analogues in patients with negative HBeAg and undetectable viral DNA at the end of treatment

\begin{tabular}{lll}
\hline All $(N=140)$ & & \\
\hline & First year & Second year \\
\hline Virological relapse & $62.0 \%(95 \% \mathrm{Cl}, 53.5-70.6 \%)$ & $79.2 \%(95 \% \mathrm{Cl}, 70.9-86.4 \%)$ \\
Clinical relapse & $28.4 \%(95 \% \mathrm{Cl}, 21.2-37.5 \%)$ & $42.9 \%(95 \% \mathrm{Cl}, 34.1-52.8 \%)$ \\
\hline
\end{tabular}


of HBsAg and risks of relapse (Fig. 2). An increase in the serum level of HBsAg following NA cessation was significantly correlated with a higher risk of subsequent virological $(P=0.00017)$ and clinical $(P=0.012)$ relapses. Moreover, no obvious nonlinearity was observed in the dose-response relationship, supporting the use of a linear term in the Cox model to summarize the hazard ratio for one log increase in the HBsAg level.

\section{Multivariate-adjusted analyses for the association between time-varying HBsAg levels and off-therapy relapses}

The association between time-varying serum levels of HBsAg and off-therapy relapses was further examined through the multivariate-adjusted Cox proportional hazard analysis, which revealed that HBsAg levels, EOT age, and EOT ALT were significant predictors of both clinical and virological relapses. For virological relapse, the serum level of HBsAg, EOT age, and EOT ALT were associated with adjusted hazard ratios of 2.10 per log $\mathrm{IU} / \mathrm{mL}$ (95\% CI, 1.45-3.04), 1.04 per year (95\% CI, 1.021.06), and 1.02 per U/L (95\% CI, 1.01-1.02), respectively (Table 3). Regarding clinical relapse, the serum level of HBsAg, EOT age, and EOT ALT were associated with adjusted hazard ratios of 2.32 per $\log \mathrm{IU} / \mathrm{mL}(95 \% \mathrm{CI}$, $1.28-4.21), 1.03$ per year (95\% CI, 1.00-1.06), and 1.03 per U/L (95\% CI, 1.02-1.05), respectively (Table 4). Furthermore, the association of HBsAg levels with outcomes did not vary with time $(P=0.19$ for virological and 0.71 for clinical relapses), thus satisfying the proportional hazard assumption for the model.

The predictive value of low HBsAg was demonstrated by 19 patients whose respective HBsAg levels ever dropped below $10 \mathrm{IU} / \mathrm{mL}$ during the off-therapy followup. Among them, only one and three patients subsequently developed clinical and virological relapses,
Table 3 Multivariate Cox porportional hazard model for virological relapse with off-therapy $\mathrm{HBsAg}$ level as a time-varying variable

\begin{tabular}{llll}
\hline Variables & Adjusted HR & $95 \% \mathrm{Cl}$ & $P$ \\
\hline Time-varying HBsAg level, log IU/mL & 2.10 & $1.45,3.04$ & $<0.0001$ \\
Male sex & 1.33 & $0.58,3.05$ & 0.49 \\
EOT age, year & 1.04 & $1.02,1.06$ & 0.0005 \\
EOT ALT, U/L & 1.02 & $1.01,1.02$ & $<0.0001$ \\
EOT AFP, ng/mL & 1.06 & $0.96,1.16$ & 0.24 \\
EOT anti-HBe-seropositive & 0.46 & $0.16,1.29$ & 0.14 \\
Pretreatment viral DNA, log IU/mL & 1.06 & $0.90,1.25$ & 0.52 \\
Pretreatment HBeAg-positive & 2.58 & $0.53,12.50$ & 0.24 \\
Pretreatment anti-HBe-positive & 4.02 & $0.88,18.3$ & 0.07 \\
\hline
\end{tabular}

Notes. AFP alpha-fetoprotein, ALT Alanine transaminase, $C /$ confidence interval, EOT end-of-therapy, $H B e A g$ hepatitis $B$ e antigen, $H B s A g$ hepatitis $B$ surface antigen, $H R$ hazard ratio

respectively (both $P<0.0001$ ), compared with those whose HBsAg levels never fell below10 IU/mL.

\section{Discussion}

In our recent study, we demonstrated that the serum gradient of HBsAg measured at the end of treatment, as well as ALT levels and age, stratified the risks of both virological and clinical relapses [20]. The current study further extends the predictive value of $\mathrm{HBsAg}$ measurement beyond the time point at NA cessation. We demonstrated that the off-therapy HBsAg level as a time-varying predictor was associated with both clinical and virological relapses in EOT HBeAg-negative patients with $\mathrm{CHB}$. Eextremely low incidence rates of clinical relapse were observed in those whose HBsAg levels fell below $10 \mathrm{IU} / \mathrm{mL}$, indicating the clinical application of HBsAg surveillance in patients attempting NA discontinuation. Therefore, monitoring serum levels of HBsAg
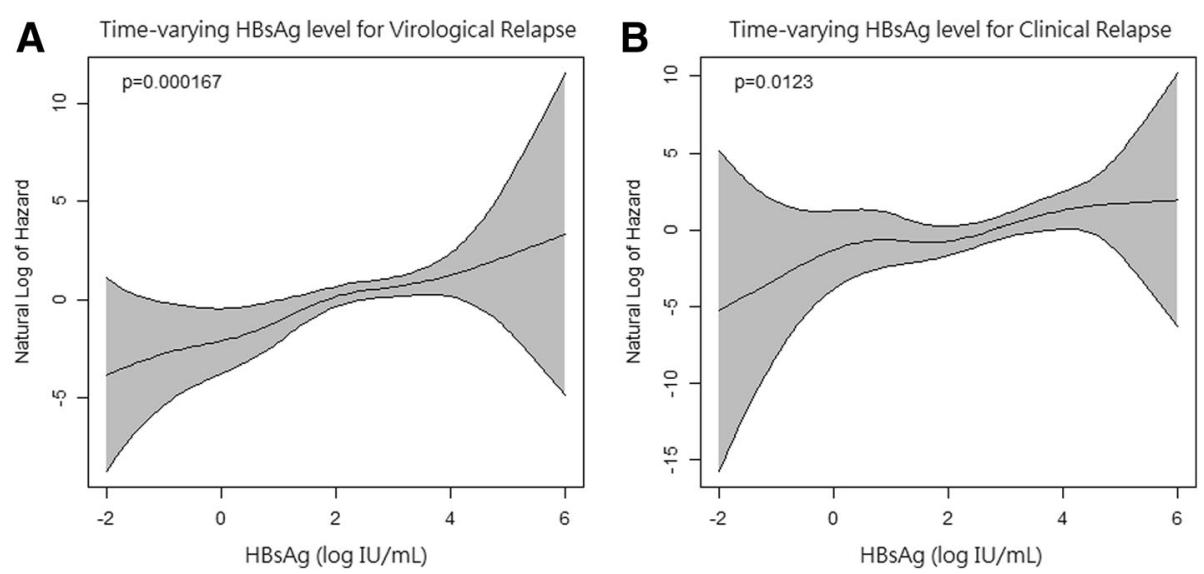

Fig. 2 Dose-response relationship for the association of off-therapy HBsAg levels with virological relapse (a) and clinical relapse (b). The dose-response curves characterized by penalized splines in the Cox model depict a significantly increasing trend; that is, increases in HBsAg levels are associated with increased risks of virological $(P=0.00017)$ and clinical $(P=0.012)$ relapses.Overall, no obvious nonlinearity was observed in the dose-response relationship, supporting the use of a linear term in the Cox model to summarize the hazard ratio for one log increase of HBsAg level 
Table 4 Multivariate Cox porportional hazard model for clinica relapse with off-therapy $\mathrm{HBsAg}$ level as a time-varying variable

\begin{tabular}{llll}
\hline Variables & Adjusted HR & $95 \% \mathrm{Cl}$ & $P$ \\
\hline Time-varying HBsAg level, log IU/mL & 2.32 & $1.28,4.21$ & 0.005 \\
Male sex & 1.05 & $0.33,3.29$ & 0.94 \\
EOT age, year & 1.03 & $1.00,1.06$ & 0.045 \\
EOT ALT, U/L & 1.03 & $1.02,1.05$ & $<0.0001$ \\
EOT AFP, ng/mL & 0.88 & $0.67,1.15$ & 0.34 \\
EOT anti-HBe-seropositive & 1.27 & $0.14,11.60$ & 0.84 \\
Pretreatment viral DNA, log IU/mL & 1.03 & $0.79,1.36$ & 0.81 \\
Pretreatment HBeAg-positive & 0.46 & $0.66,3.21$ & 0.43 \\
Pretreatment anti-HBe-positive & 0.47 & $0.70,3.20$ & 0.44 \\
\hline
\end{tabular}

Notes. AFP alpha-fetoprotein, ALT Alanine transaminase, $C l$ confidence interval, EOT end-of-therapy, $H B e A g$ hepatitis $B$ e antigen, $H B s A g$ hepatitis $B$ surface antigen, $H R$ hazard ratio

may guide the selection of patients who can maintain sustained viral suppression.

Inactive carriers have been found to have low $\mathrm{HBsAg}$ levels [23-25], with HBsAg levels $<1000 \mathrm{U} / \mathrm{mL}$ having an $87.9 \%$ positive predictive value and $96.7 \%$ negative predictive value for patients with HBV DNA <2000 IU/ mL. A recent study [21] in Northern Taiwan involving 117 patients receiving entecavir treatment reported that serum HBV DNA levels at 3 and 6 months off-therapy were associated with clinical relapse, and the HBsAg level at 6 months off-therapy had a weak association with clinical relapse. Furthermore, $13.6 \%$ of the patients had sustained virological response (undetectable HBV DNA levels at 12 months off -therapy). No clinical relapse was observed during a mean 24.8-month followup, suggesting that patients without HBsAg clearance could have sustained clinical and viral remission. However, EOT HBsAg was the only factor associated with sustained virological response $(P=0.009)$. Another study involving 252 patients in southern Taiwan [26] also showed that the combination of age $(<55$ years $)$ and EOT HBsAg levels $(<150 \mathrm{IU} / \mathrm{mL})$ was associated with a low rate of virologic relapse. The decline level of HBsAg during treatment was higher in patients with $\mathrm{HBsAg}$ clearance than in those without HBsAg clearance. However, both studies mainly relied on EOT HBsAg levels to predict outcomes, and the association of HBsAg kinetics and relapse risk was not discussed. Our study is the first to discuss HBsAg as a time-varying factor for durable remission.

Although patients subjected to NA treatment had a favorable response with regard to HBV DNA levels, the observed HBsAg decline in such patients was lower than in those treated with interferon. This is probably due to the mechanism of NA treatment. NA treatment affects the reverse transcription of pregenomic RNA, but it does not affect covalently closed circular DNA and subgenomic RNA, which have translational activities associated with HBsAg levels [27]. Moreover, serum HBsAg levels reflect active intrahepatic covalently closed circular DNA and have additional value as markers of ontreatment efficacy [28]. However, the NA potentially restores immune responses [29], which possibly results in HBsAg decline. This hypothesis is supported by data that HBsAg decline was strong in patients with higher ALT at the baseline [30] or higher levels of interferon gamma-induced protein 10 at the baseline, which indicates a reasonable level of immune response in such patients. Interferon gamma-induced protein 10 is a chemokine, which is a response to interferon gamma and may reflect the innate immune response against HBV [31]. Our data also show that the serum gradient of HBsAg was associated with the subsequent occurrence of virological and clinical relapses. Especially, the patients, whose HBsAg levels below $10 \mathrm{IU} / \mathrm{mL}$ not only at the end of therapy but along the off-therapy follow-up, had very low risk of relapses. Therefore, HBsAg kinetics may be a useful means to monitor NA treatment.

In the current strategies of NA-off follow-up, the HBV DNA and ALT would be monitored routinely and closely. However, elevation of ALT is usually a late event preceded by viral rebound and cannot be regarded as an early predictor. Following abrupt resurgence of viremia, severe acute exacerbation could rapidly ensue and incur the risk of liver failure. Besides, measurement of viral DNA remains costly. Our study showed the HBsAg kinetics has a linear association with relapse risks. Therefore, HBsAg level monitoring, every three months for instance, provides a chance to identify those who may not need frequent viral DNA measurement, and implicates a safe follow-up strategy with less expense.

This study has several strengths. First, we monitored HBsAg levels during the entire follow-up instead of at a single time point. Second, this research was conducted in a real-world setting that reflects "in-field" practice. For external validity, patients were recruited from multiple sites and treatment was not restricted to a single NA. Third, a minimum of 3-year NA therapy enabled most patients to obtain remission status before discontinuing NAs. Finally, stringent monitoring with an interval of 3 months enabled us to observe both virological and clinical relapses closely.

Several limitations were noted in this study. First, because this study prospectively started after NA discontinuation, blood samples prior to the baseline were unavailable. Therefore, we could not ascertain when HBV DNA became undetectable during the treatment by using a standardized protocol. We acknowledge that this is a major limitation because the length of consolidation following remission of viremia has been shown to influence the risk of relapse 
[14]. Nonetheless, this limitation is unlikely to bias our finding regarding the dose-response association of timevarying HBsAg with off-therapy relapses. Third, we could not quantify pretreatment HBsAg levels exceeding $250 \mathrm{IU} /$ $\mathrm{mL}$ and were thus unable to calculate the exact decline of HBsAg during therapy. Intriguingly, a recent small study in Spain suggested that an on-therapy HBsAg decline of more than $5000 \mathrm{IU} / \mathrm{mL}$ might indicate durable remission [32]. Finally, because our study exclusively recruited Asian patients whose viral genotype was either type B or C [33], extrapolation to the Western population requires validation.

\section{Conclusions}

In conclusion, this study reveals a dose-response association between time-varying $\mathrm{HBsAg}$ and off-therapy relapse following the cessation of NA therapy in patients with $\mathrm{CHB}$. By reporting a negligible risk of subsequent relapse in patients whose HBsAg dropped to a considerably low level $(<10 \mathrm{IU} / \mathrm{mL})$, we demonstrate the potential utility of monitoring $\mathrm{HBsAg}$ levels in patients with $\mathrm{CHB}$ who discontinue NA treatment. These findings may not only implicate a safe strategy to monitor patients who attempt NA cessation but also stimulate further research to elucidate the clinical relevance of HBsAg quantification.

\section{Abbreviations}

ALT: Alanine transaminase; CHB: Chronic hepatitis B; Cl: Confidence interval; EOT: End of therapy; HBeAg: Hepatitis B envelope antigen; HBsAg: Hepatitis B surface antigen; HBV: Hepatitis B virus; IQR: Interquartile range; NA: Nucleos(t)ide analog

\section{Acknowledgements}

The authors are indebted to our colleagues involved in the clinical care of these patients. We also appreciate the efforts by Ms. Ying-Ju Lee, Ms. Hsin-Yi Tsai, and Ms. Tzu-En Tsai.

\section{Grant support}

Cathay General Hospital and Fu-Jen University(104-GGH-FJU-11), E-Da Hospital (EDAHP105019), and the Taipei Pathology Institutes (TIP104-2).

\section{Availability of data and materials}

The research datasets are not able to deposit in publicly available repositories or present in the main paper. The regulations of IRB in our hospital (E-Da hospital, Kaohsiung, Taiwan) strictly prohibit public spread of these data in order to protect patient privacy. The corresponding author (Yao-Chun Hsu: gatsbyhsu@yahoo.com.tw) confirms that the data will be available upon request.

\section{Authors' contributions}

GUARANTOR OF THE ARTICLE: Y-CH. Concept and design: YCH, Y-TH, and J-TL Data collection: Y-CH, C-YC, M-SW, T-HY, L-RM, C-MT, and C-WL. Data analysis and interpretation: N-HC, Y-TH, C-YW, Y-CH, and J-HK. Manuscript drafting: N$\mathrm{HC}$, and $\mathrm{Y}-\mathrm{CH}$. Edition and revision of the manuscript: All authors. All authors read and approved the final manuscript.

\section{Ethics approval and consent to participate}

Institutional review boards approved the study protocol (EMRP100-049) in all hospitals for patient recruitment and database establishment. Data analysis specifically for this study was also approved (EMRP-104-082). Written informed consent was obtained from all participants prior to their enrollment.
Consent for publication

Not applicable.

\section{Competing interests}

YCH has received lecture fees from Bristol-Myers Squibb, Roche, and Gilead Sciences. JHK is a consultant for Abbott, Abbvie, Bayer, Boehringer Ingelheim, Bristol-Myers Squibb, Gilead Sciences, GlaxoSmithKline, Johnson \& Johnson, Merck Sharp \& Dohme, Novartis, and Roche and is on the speaker's bureau for Abbott, Roche, Bayer, Bristol-Myers Squibb, GlaxoSmithKline, and Novartis. $J T L$ has received a research grant from Gilead Sciences in another unrelated study. All other authors have no competing interests to declare.

\section{Publisher's Note}

Springer Nature remains neutral with regard to jurisdictional claims in published maps and institutional affiliations.

\section{Author details}

${ }^{1}$ Cathay General Hospital, Taipei, Taiwan. ${ }^{2}$ School of Medicine, Fu Jen Catholic University, New Taipei, Taiwan. ${ }^{3}$ Institute of Statistical Science, Academia Sinica, Taipei, Taiwan. ${ }^{4}$ Division of Gastroenterology, Taichung Veterans General Hospital, Taichung, Taiwan. ${ }^{5}$ Faculty of Medicine, School of Medicine, National Yang-Ming University, Taipei, Taiwan. ${ }^{6}$ Division of Gastroenterology, Fu-Jen Catholic University Hospital, New Taipei, Taiwan. ${ }^{7}$ Department of Internal Medicine, National Taiwan University Hospital, Taipei, Taiwan. ${ }^{8}$ Graduate Institute of Clinical Medicine, National Taiwan University, Taipei, Taiwan. ${ }^{9}$ Department of Internal Medicine, Tainan Municipal Hospital, Tainan, Taiwan. ${ }^{10}$ Division of Gastroenterology, E-Da Hospital/I-Shou University, Kaohsiung, Taiwan. ${ }^{11}$ Department of Internal Medicine, Lotung Poh-Ai Hospital, Yilan Country, Taiwan. ${ }^{12}$ Sijhih Cathay General Hospital, New Taipei, Taiwan. ${ }^{13}$ No.510, Zhongzheng Rd., Xinzhuang Dist, New Taipei City 24205, Taiwan.

Received: 7 February 2017 Accepted: 20 November 2017

Published online: 08 December 2017

References

1. Liaw YF, Chu CM. Hepatitis B virus infection. Lancet. 2009;373:582-92.

2. El-Serag HB. Epidemiology of viral hepatitis and hepatocellular carcinoma. Gastroenterology. 2012;142:1264-73.

3. European Association For The Study Of The L. EASL clinical practice guidelines: Management of chronic hepatitis B virus infection. J Hepatol. 2012:57:167-85.

4. Lok AS, McMahon BJ. Chronic hepatitis B: update 2009. Hepatology. 2009;50:661-2.

5. Liaw YF, Kao JH, Piratvisuth $\mathrm{T}$, et al. Asian-Pacific consensus statement on the management of chronic hepatitis B: a 2012 update. Hepatol Int 2012;6:531-61.

6. Lin $\mathrm{CL}$, Kao JH. Recent advances in the treatment of chronic hepatitis $\mathrm{B}$. Expert Opin Pharmacother. 2011;12:2025-40.

7. Chang TT, Liaw YF, SS W, et al. Long-term entecavir therapy results in the reversal of fibrosis/cirrhosis and continued histological improvement in patients with chronic hepatitis B. Hepatology. 2010;52:886-93.

8. Marcellin P, Gane E, Buti M, et al. Regression of cirrhosis during treatment with tenofovir disoproxil fumarate for chronic hepatitis B: a 5-year openlabel follow-up study. Lancet. 2013;381:468-75.

9. CY W, Lin JT, Ho HJ, et al. Association of nucleos(t)ide analogue therapy with reduced risk of hepatocellular carcinoma in patients with chronic hepatitis B: a nationwide cohort study. Gastroenterology. 2014;147:143-51.

10. Chiang CJ, Yang YW, Chen JD, et al. Significant reduction in end-stage liver diseases burden through the national viral hepatitis therapy program in Taiwan. Hepatology. 2015;61:1154-62.

11. Reijnders JG, Perquin MJ, Zhang N, et al. Nucleos(t)ide analogues only induce temporary hepatitis B e antigen seroconversion in most patients with chronic hepatitis B. Gastroenterology. 2010;139:491-8.

12. Chaung $\mathrm{KT}, \mathrm{Ha} \mathrm{NB}$, Trinh $\mathrm{HN}$, et al. High frequency of recurrent viremia after hepatitis B e antigen seroconversion and consolidation therapy. J Clin Gastroenterol. 2012:46:865-70.

13. Tseng TC, Liu CJ, TH S, et al. Young chronic hepatitis B patients with nucleos(t)ide analogue-induced hepatitis $B$ e antigen seroconversion have a higher risk of HBV reactivation. J Infect Dis. 2012;206:1521-31. 
14. Chi H, Hansen BE, Yim C, et al. Reduced risk of relapse after long-term nucleos(t)ide analogue consolidation therapy for chronic hepatitis B. Aliment Pharmacol Ther. 2015;41:867-76.

15. Fung J, Lai CL, Yuen J, et al. Randomized trial of lamivudine versus entecavir in entecavir-treated patients with undetectable hepatitis B virus DNA: outcome at 2 Years. Hepatology. 2011;53:1148-53.

16. Chen $\mathrm{CH}, \mathrm{SN}$ L, Hung $\mathrm{CH}$, et al. The role of hepatitis B surface antigen quantification in predicting HBsAg loss and HBV relapse after discontinuation of lamivudine treatment. J Hepatol. 2014;61:515-22.

17. Chan HL, Wong GL, Chim AM, et al. Prediction of off-treatment response to lamivudine by serum hepatitis B surface antigen quantification in hepatitis B e antigen-negative patients. Antivir Ther. 2011;16:1249-57.

18. Seto WK, Hui AJ, Wong WW, et al. Treatment cessation of entecavir in Asian patients with hepatitis B e antigen negative chronic hepatitis B: a multicentre prospective study. Gut. 2015;64:667-72.

19. Jeng WJ, Sheen IS, Chen YC, et al. Off-therapy durability of response to entecavir therapy in hepatitis B e antigen-negative chronic hepatitis B patients. Hepatology. 2013;58:1888-96

20. Hsu YC, Mo LR, Chang CY, et al. Association between serum level of hepatitis $B$ surface antigen at end of entecavir therapy and risk of relapse in E antigen-negative patients. Clin Gastroenterol Hepatol. 2016;14:1490-8.

21. Wang CC, Tseng KC, Hsieh TY, et al. Assessing the durability of Entecavirtreated hepatitis B using quantitative HBsAg. Am J Gastroenterol. 2016;111: 1286-94.

22. Papatheodoridis G, Vlachogiannakos I, Cholongitas E, et al. Discontinuation of oral antivirals in chronic hepatitis B: A systematic review. Hepatology. 2016;63:1481-92

23. Jaroszewicz J, Calle Serrano B, Wursthorn K, et al. Hepatitis B surface antigen ( $\mathrm{HBs} A g$ ) levels in the natural history of hepatitis B virus (HBV)-infection: a European perspective. J Hepatol. 2010;52:514-22.

24. Martinot-Peignoux M, Lada O, Cardoso AC, et al. Quantitative HBsAg: a new specific marker for the diagnosis of HBsAg inactive carriage. Hepatology. 2010;52:992

25. Yakut M, Bektas M, Seven $G$, et al. Characterization of the inactive HBsAg carrier state with 3 year follow-up. J Hepatol. 2011;54:S159.

26. Chen $\mathrm{CH}$, Hung $\mathrm{CH}$, Hu TH, et al. Association between level of hepatitis $\mathrm{B}$ surface antigen and relapse after entecavir therapy for chronic hepatitis B virus infection. Clin Gastroenterol Hepatol. 2015;13:1984-92.

27. Manesis EK, Papatheodoridis GV, Tiniakos DG, et al. Hepatitis B surface antigen: relation to hepatitis $B$ replication parameters in HBeAg-negative chronic hepatitis B. J Hepatol. 2011;55:61-8.

28. Chan HL, Wong WW, Tse AM, et al. Serum hepatitis B surface antigen quantitation can reflect hepatitis B virus in the liver and predict treatment response. Clin Gastroenterol Hepatol. 2007;5:1462-8.

29. Boni C, Laccabue D, Lampertico P, et al. Restored function of HBV-specific T Cells after long-term effective therapy with nucleos(t)ide analogues. Gastroenterology. 2012;143:963-73.

30. Zoutendijk R, Hansen BE, van Vuuren AJ, et al. Serum HBsAg decline during long-term potent nucleos(t) ide analogue therapy for chronic hepatitis $B$ and prediction of HBsAg loss. J Infect Dis. 2011:204:415-8.

31. Jaroszewicz J, Ho H, Markova A, et al. Hepatitis B surface antigen ( $\mathrm{HBs} A \mathrm{Ag}$ ) decrease and serum interferon-inducible protein-10 levels as predictive markers for HBsAg loss during treatment with nucleoside/nucleotide analogues. Antivir Ther. 2011;16:915-24.

32. Buti M, Casillas R, Riveiro-Barciela M, Homs M, Tabernero D, Salcedo MT, Rodriguez-Frias F, Esteban R. Tenofovir discontinuation after long-term viral suppression in $\mathrm{HBeAg}$ negative chronic hepatitis B. Can HBsAg levels be useful? Clin Virol. 2015;68:61-8.

33. Kao JH, Chen PJ, Lai MY, et al. Hepatitis B genotypes correlate with clinical outcomes in patients with chronic hepatitis B. Gastroenterology. 2000;118:554-9.

\section{Submit your next manuscript to BioMed Central and we will help you at every step:}

- We accept pre-submission inquiries

- Our selector tool helps you to find the most relevant journal

- We provide round the clock customer support

- Convenient online submission

- Thorough peer review

- Inclusion in PubMed and all major indexing services

- Maximum visibility for your research

Submit your manuscript at www.biomedcentral.com/submit

CBiomed Central 